\title{
Application of Digital Virtual Tour Technology in Intelligent Scenic Area Design
}

\author{
Fang Shiqiao ${ }^{1,2}$ \\ ${ }^{1}$ Guangxi Teachers Education University, Nanning, 530001, P.R. China \\ ${ }^{2}$ Research Center of new urbanization in Minority Areas \\ Xuexi123@163.com
}

Keywords: Virtual tourism, new-type urbanization, Social governance, Intelligent scenic spot, Finite element, Intelligent, Java3D, Creator.

\begin{abstract}
The intelligent tourism service is the core content of the intelligent travel, and it is also the key link of future tourism industry changing to the modern service industry. In order to design reasonable intelligent scenic scenes and tourist paths, this paper proposes a new digital virtual design method of intelligent tour scenic area, and uses the finite element method to design mathematical model of scenic 3D modeling. This paper also designs the corresponding algorithm, based on Java3D and Creator software of Web server, and designs 3D scenic spot, so that visitors can experience area through the form of digitized virtual, which improves the digital and intelligent level of service area and provides the theory reference for the innovative design of the scenic tour.
\end{abstract}

\section{Introduction}

As a tourist service bearing body, tourism enterprises, how to face the development opportunity brought by the intelligent tourism and realize intelligent upgrade is an urgent problem to be solved. At present the main VR browsers are VRML, Cult3D, Java3D, which is used to construct the virtual tourism space and achieve real-time online watch browse. Java3D modeling includes 3D geometric objects, colors, materials, lighting, camera, animation and sensor of providing interactive. 3D technology can easily create 3D model scene real-time on the internet $[1,2]$. This paper uses the Web server technology and Java3D to do digital virtual design on the scenic area, including real selection, modeling and scenic roaming. The main flow is as shown in Figure 1.

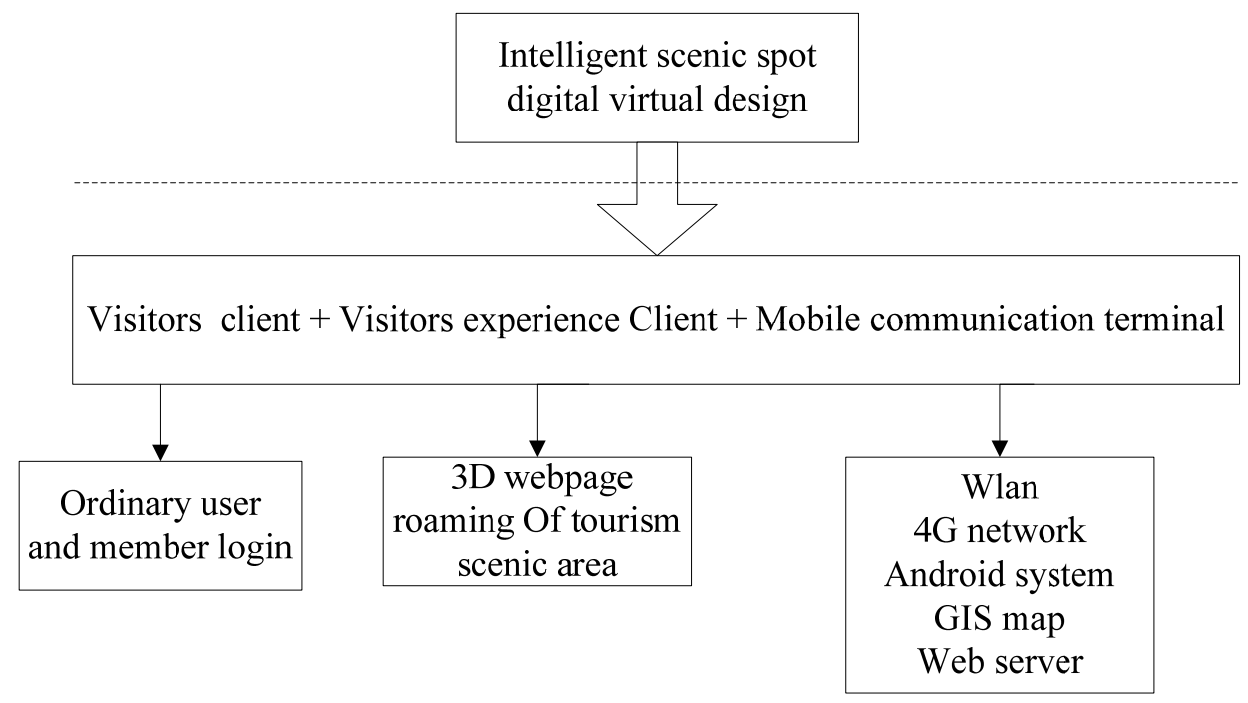

Fig. 1 Digital virtual design framework of intelligent area

Figure 1 shows the digital virtual design framework of the scenic tour [3]. Around social, localization and mobile internet search engine, this paper designs virtual tour scenic area of digital, virtual and intelligent, and it can provide $3 \mathrm{D}$ webpage roaming scene as the user experience, combining the mobile communication terminal, and provide users with more convenient service. 
With the accelerated development of the tourism industry, the confliction of city land use is larger. Land use widespread expands faster, land use is inefficient and the land utilization benefit is imbalance. How to improve land use benefits, particularly realizing the balanced development of social economic and environmental benefits, it is an inevitable requirement for sustainable development and the construction of ecological civilization society.

\section{The Scenic Spots 3D Modeling based on Finite Element Method}

3D modeling of the scenic tour using the finite element method is mainly based on the position change in the modeling process [4-6]. The finite element mathematical model can modeling through coordinate differential element method. Any position of scenic spots can be expressed as:

$$
\beta(a, b, c)=d \cdot \Delta \alpha-(a-1) \frac{2 \pi}{M}\left[(e-1) d r+\frac{d r}{2}\right] \frac{\tan \theta}{z} .
$$

$a$ is the building material position, $b$ represents the angle increment, $c$ represents the material number, $M$ is the model number, $\theta$ is a architecture design view, $d r$ is the modeling thickness, $z$ is the path for the scenic area scenic modeling. The mapping of each element can be expressed as:

$$
\left\{\begin{array}{l}
d G x(a, b, c)=J x 1 l(a, b, c) d r+J x 2 \frac{d r}{\cos \theta} \\
d G y(a, b, c)=J y 1 l(a, b, c) d r+J y 2 \frac{d r}{\cos \theta} . \\
d G z(a, b, c)=J z 1 l(a, b, c) d r+J z 2 \frac{d r}{\cos \theta}
\end{array}\right.
$$

Among them, $d G x(a, b, c), d G y(a, b, c)$ and $d G z(a, b, c)$ are partial differential of mapping function in three directions, $J x 1$ is the longitudinal mapping coefficients in three directions, $J x 2$ is the horizontal mapping coefficients in the three directions, $l(a, b, c)$ is building cover thickness, $l(a, b, c) d r$ is the construction layer covering the area.

$$
l(a, b, c) d r=\left\{\begin{array}{c}
f r \cos (\beta(a, b, c)) \\
0
\end{array} \quad \beta(a, b, c) \in[\psi 1, \psi 2] .\right.
$$

Through the coordinate transform method, the space infinitesimal coordinate can be transferred to the scenic area, and the transformation matrix is:

$$
\left[\begin{array}{l}
d G s(a, b, c) \\
d G d(a, b, c) \\
d G w(a, b, c)
\end{array}\right]=\left[\begin{array}{l}
d G x(a, b, c) \\
d G y(a, b, c) \\
d G z(a, b, c)
\end{array}\right] \cdot T \text {. }
$$

The value of $T$ can be substituted into:

$$
\left\{\begin{array}{c}
d G s(a, b, c)=d G \times 1(a, b, c) \cos \beta(a, b, c)+d G \times 2(a, b, c) \sin \beta(a, b, c) \\
d G d(a, b, c)=d G y(a, b, c) \sin \beta(a, b, c)-d G y 2(a, b, c) \cos \beta(a, b, c) . \\
d G w(a, b, c)=-d G z(a, b, c)
\end{array}\right.
$$

The finite element model of the whole scenic spot can be expressed as: 


$$
\left\{\begin{array}{l}
G x(b)=\sum_{i=1}^{M} \sum_{u=1}^{N} d G x(a, b, c) \\
G y(b)=\sum_{i=1}^{M} \sum_{u=1}^{N} d G y(a, b, c) . \\
G z(b)=\sum_{i=1}^{M} \sum_{u=1}^{N} d G z(a, b, c)
\end{array}\right.
$$

Among them, $G x(b), G y(b)$ and $G z(b)$ are the total mapping function of three axial, $N$ is mapping infinitesimal. Through different texture mapping, it can construct the general picture of wisdom scenic area, and use Java to define the dynamic and static objects of scenic area [7]. The main procedures are as follows:

public class GameMain \{

private static int scrWidth $=500$;

private static int scrHeight $=300$;

private static int scrBitdepth $=82$;

private JFrame gameFrame;

private WrapCheckers3D wrap;

public static void main(String[] args)

\{

GameMain game = new GameMain();

\}

public GameMain()

$\{$ ScreenManager screen $=$ new ScreenManager(scrWidth,scrHeight,scrBitdepth,"Java 3D Test ");

screen.setWindowMode();

gameFrame $=$ screen.getFrame () ;

wrap $=$ new WrapCheckers3D(scrWidth,scrHeight $)$;

gameFrame.add(wrap);

\}

\section{Intelligent Scenic Spot Design based on Virtual Tourism}

In order to effectively verify the effectiveness and reliability of algorithm designed in second section, this paper uses Java3D and Creator software to do digital virtual design on intelligent tourism [8-10]. Firstly it uses Java3D to establish the virtual scene as shown in Figure 2.

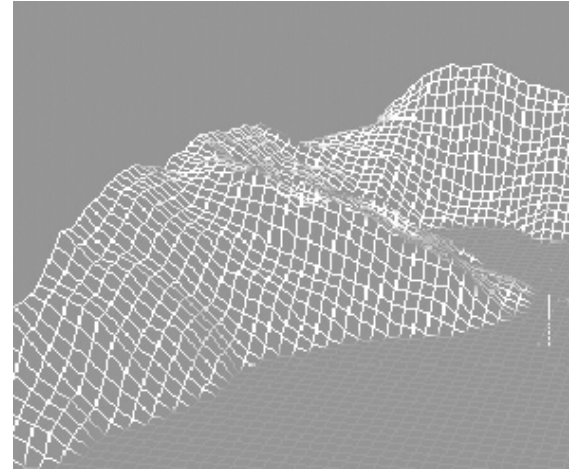

Fig. 2 Design of Javd3D scene modeling

In the Java3D scene graph, it contains the nodes of constructing 3D terrain model, scene interaction, Shape3D, Appearance and Geometry model describing geometry properties and texture attributes, which are used to build the objects not in the real map [11]. In the actual design, it can first select the real as the design basis, as shown in Figure 3. 


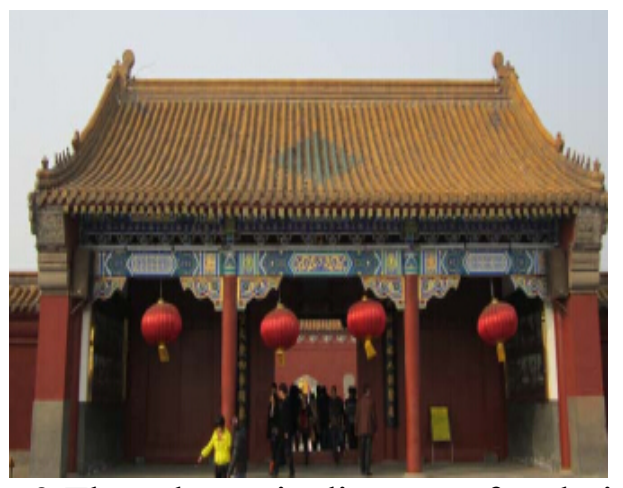

Fig. 3 The schematic diagram of real view

As shown in Figure 3, in the construction of the visual model, there are often redundant data phenomenon inherent, redundant polygon generally exits the internal model, such as the bottom surface of building, the inner wall, and the floor connecting surface [12]. Imported into the Creator software is as shown in Figure 4.

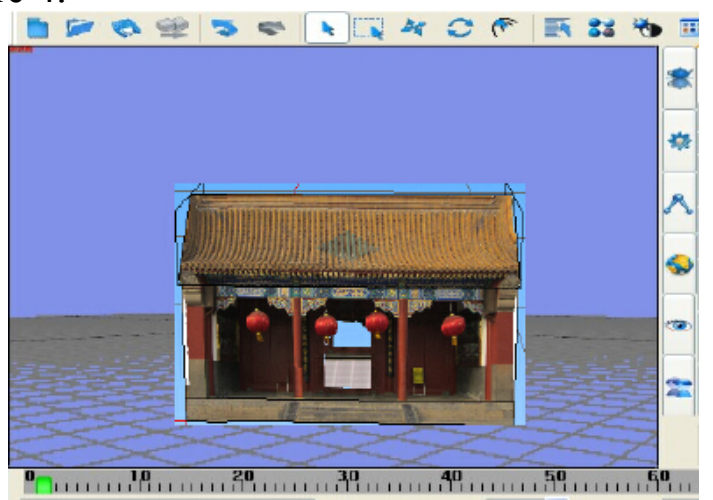

Fig. 4 The hotel service brand internal constraint relation

After the real map is imported into Creator, the landscape can be re-layout and designed, and Creator can also do texture mapping [13]. Texture mapping is the 2D texture pixel in texture space is mapped into 3D shape, and is projected onto the screen space.

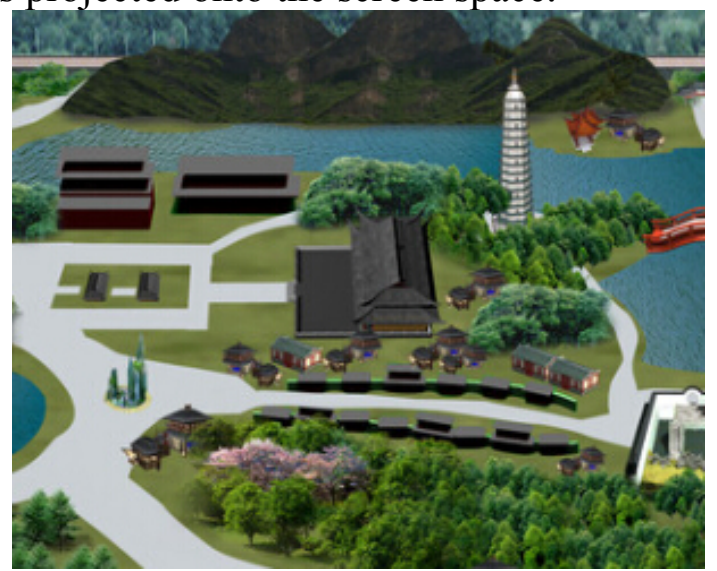

Fig. 5 The 3D virtual scenic spot

Figure 5 shows the schematic diagram of the overall design virtual scenic spot. The use of Web3D technology can design the scenic into a 3D map form, and visitors can be designed into a virtual human [14]. In order to improve the visitor experience, it can use the virtual people roaming form to visit the virtual scenic spot. Through the analysis of the coupling result, the coupling model can effectively calculate the coupling degree of ecological benefits and social benefits, which provides a theoretical basis for the research on the evaluation of land use efficiency. 


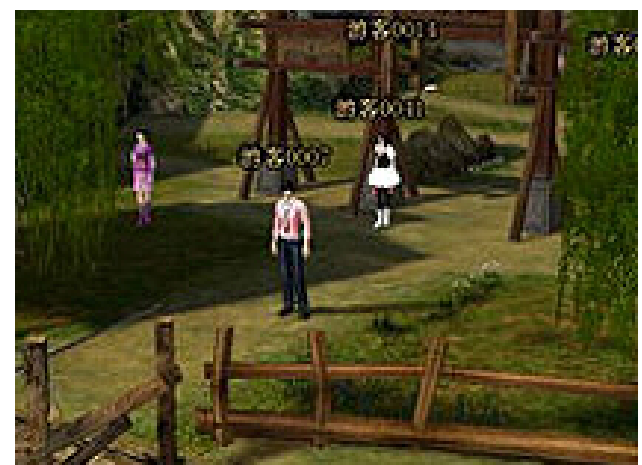

Fig. 6 Schematic diagram of virtual scene roaming

Figure 6 shows the tour scenic spot diagram of virtual scene roaming. So the visitors can fully experience the scene in the scenic spot before visiting, so as to choose the sites of interest to visit, which improves the intelligent level of service area [15]. The business can also be applied to the mobile phone terminal, using the mobile phone Web server and $4 \mathrm{G}$ wireless network, combined with GIS map, and give full play to the role of digital technology in the tourism services, which can provide convenient services for tourists.

\section{Summary}

For the needs of intelligent tour scenic area digital and intelligent service, this paper designs a new virtual tour scenic area, combined with finite element thought, establishes the function of scenic model and texture mapping, and through the Java3D programming to achieve the algorithm. This paper uses the Java3D and Creator software to do virtual design on the intelligent scenic spot, and uses Web server to develop the scenic spot Webpage roaming experience model, so that visitors can experience the true feelings of scenic area through the internet. The study has not be is not verified on mobile WLAN and 4G signal. In future research it can develop the experience system suitable for mobile phone, to further improve the convenience of the system.

\section{Acknowledgements}

Research Center of open fund project "Ethnic Villages Tourism Destination Residents living condition assessment and strategy optimization" (serial number: 2014YB008) in minority areas of new towns.Fund project: General project of the new research center is open fund Urbanization in Ethnic Areas :"Ethnic Villages Tourism Destination Residents living condition assessment and strategy optimization".(No: 2014YB008)

\section{References}

[1] L.Y. Zhang, N. Li, M. Liu. The basic concept and theoretical system of wisdom tourism. Journal of travel, 2012, 4(5): 32-35.

[2] L.Y. Zhang. The wisdom tourism: customization and intelligent public service era. Travel journal, 2012, 4(2): 62-65.

[3] W.D. Jin. The wisdom tourism and the construction of public service system. Journal of travel, 2012, 3(2): 23-25.

[4] Z. Zhu, X. Zhang. Construction of the wisdom of tourism perception system and management platform. Journal of Jiangsu University (SOCIAL SCIENCE EDITION), 2013, 4(6): 35-38.

[5] G.P. Deng, Z.F. Shao. Jiuzhaigou wisdom scenic area management and service based on video cruise. Computer engineering and design, 2012, 4(11): 63-65.

[6] J.L. Liu, Y.F. Fan. The value, construction and development trend of wisdom tourism. Chongqing social science, 2012, 5(10): 54-55. 